\title{
Genetic resources in creating sustainable diseases of introgressive spring wheat forms
}

\author{
Abugaliyeva A.I. ${ }^{1 *}$, Morgounov A.I. ${ }^{2}$, Kozhakhmetov K. ${ }^{1}$, Chudinov V.A. ${ }^{3}$, \\ Shamanin V.P. ${ }^{4}$, Gultyaeva E.N. ${ }^{5}$, Kolomiyec T.M. ${ }^{6}$, Rsaliyev A.Sh. ${ }^{7}$, Salina E.A. ${ }^{8}$ \\ ${ }^{1}$ Kazakh Research Institute of Agriculture and Plant Growing, Almalybak, Kazakhstan \\ ${ }^{2}$ CIMMYT, Turkey \\ ${ }^{3}$ Karabalyk Agricultural Experimental Station, Kostanay region, Kazakhstan \\ ${ }^{4}$ Omsk State Agrarian University, Omsk, Russia \\ ${ }^{5}$ All-Russian Institute of Plant Protection, St. Petersburg, Russia \\ ${ }^{6}$ All-Russian Research Institute of Phytopathology, Moscow, Russia \\ ${ }^{7}$ Research Institute for Biological Safety Problems, Zhambyl region, Kazakhstan \\ ${ }^{8}$ Institute of Cytology and Genetics, SB RAS, Novosibirsk \\ *e-mail:kiz_abugalieva@mail.ru
}

Introgression spring wheat forms with T. militinae, T. timopheevii, T. dicoccum, T. kiharae and T. zhykovskyi were screened for disease resistance (leaf, stem, yellow rust, septoria, mildew, smut) in: Almalybak, Karabalyk; OmSAU, Turkey; on the infectious background St. Petersburg, Otar; Moscow, Turkey; at the cytological and genetic level - KazRIAPG, ICG and ARRIPS, CIMMYT. On a natural infectious and background from 7 to 21 samples $(23-50 \%)$ were allocated for leaf rust resistance: $6583 \times$ T. timopheevii (Lr34, Lr36, Lr68), Kazakhstanskaya $10 \times$ T. dicoccum (Lr46, Lr68), 6569 × T. militinae (Lr34, Lr46), $6625 \times T$. timopheevii (Lr14, Lr46) and $6631 \times$ T. timopheevii (Lr9, Lr14) and species T. militinae (Lr19, Lr68), T. timopheevii (Lr19, Lr68) and T. kiharae (Lr68). According to stem rust, advanced lines block was immune (Sr2) and highly resistant (5-10) relative to cvs-standard Kazakhstanskaya 10 (15-75) and to T. militinae, T. timopheevii, T. kiharae Sr36. 9 synthetic spring wheat lines were found (Kazakhstanskaya $10 \times T$. dicoccum, $6569 \times$ T. millitinae-1, $6569 \times$ T. millitinae-2, $6628 \times$ T. millitinae, $6625 \times T$. timopheevii-1, $6625 \times T$. timopheevii-2, 6625 $\times$ T. timopheevii-3, 6628 $\times$ T. timopheevii-1 and $6628 \times$ T. timopheevii-2), which show horizontal rust resistance. Genotypes $6628 \times T$. militinae and $6569 \times$ T. militinae-1 (TIRS.1BL; estimated substitution $2 \mathrm{~B}(2 \mathrm{G})$ or translocation T2B-2G); $6569 \times$ T. militinae-2 (TIRS.1BL; T3GS/3BL) (I.G. Adonina, unpublished data). According to maximum yield, genotypes with brown and stem rust resistance are $6569 \times T$. militinae-2 (3.2-5.2 t/ha); 6569 $\times$ T. militinae-1 (3.0-5.7 t/ha); $6628 \times T$. timopheevii-3 (3.0-5.7 t/ha). The following genotypes were distinguished by minimum powdery mildew infection: Kazakhstanskaya $10 \times T$. timopheevii; $6631 \times$ T. timopheevii (0-5\%); 6625 $\times$ T. timopheevii-2 (10-15\%). Selected resistance forms were evaluated by hight yield, quality, DUS-test, transferred to double haploid basis and for (Tim-biday, Gunticum, VEK). 